\title{
DETERMINATION OF PRESSURE DROP IN HORIZONTAL PIPES FOR AIR - WATER TWO PHASE FLOW
}

\begin{abstract}
ALINA FILIP - Lecturer, Technical University of Civil Engineering, Faculty of Building Services, e-mail: alinafilip69@yahoo.com
\end{abstract}

\begin{abstract}
The gas-liquid two-phase flow is characterized by continuous and local change of surface separation of phases and by their mutual interactions. Due to the instability of the flow, heat transfer and mass, a precise analytical approach is difficult to achieve. Despite these difficulties, efforts are underway to progress from the more frequent empirical studies to reliable analytical models. This requires an accurate research of the processes involved in the two phase flow and how they interact with one another. This paper aims to determine the pressure drop for a two-phase flow in a horizontal pipe of a heating plant. The author compares the results obtained by numerical simulation with existing results in the domain. The mixture is air-water, at an environmental temperature of $25^{\circ} \mathrm{C}$.
\end{abstract}

Keywords: two-phase flow, air-water, pressure drop, horizontal pipe

\section{Introduction. Importance of Pressure Drop Monitoring in Pipes}

When designing a thermo-hydraulic apparatus in which a two-phase flow takes place, it is important to correctly determine the pressure drop for measuring and functioning of the aggregate in economical and technically adequate conditions. Experimental research on pressure drop occupies a large part in the literature referring to two-phase flows. Despite numerous researches in this domain, the pressure drop in a two-phase flow can be measured, in most cases, with a precision of $\pm 50 \%$, and in extreme conditions, with $\pm 100 \%$.

In the case of a two-phase flow in a pipe, the total pressure drop is calculated by taking into account the next components, expressed in the form of pressure drops (variations) [1]:

- the component that occurs due to friction, caused by phenomena taking place at the pipe wall and the friction between phases;

- the component that occurs after the acceleration of the phases due to pressure drop or change of phase;

- the component that occurs due to the difference in height of slanted or vertical pipes.

Therefore, on the unit length, the pressure variation can be expressed by the equation:

$$
\left(\frac{\Delta p}{\Delta L}\right)_{i \quad e}=\left(\frac{\Delta p}{\Delta L}\right)_{f r}+\left(\frac{\Delta p}{\Delta L}\right)_{a c c}+\left(\frac{\Delta p}{\Delta L}\right)_{h}
$$

\section{Mathematical Models Used in Pressure Drops Calculation in Flows Through Pipes}

Compared with the single-phase flow, in the case of the two-phase flow the pressure drop due to friction can be determined both from the momentum transfer at the wall and from the momentum transfer at the separation surface between the two phases. The friction between the phases usually depends on the manner of the flow and on the flow motion (boundary-layer or turbulent) of the two phases. 
There are a few empirical correlations for calculating the pressure drop due to friction in the internal two-phase flows [2]:

- the Friedel Correlation (1979) begins with a single-phase model and determines a correction factor for adjusting the energy and momentum transfer in the two-phase flow. The method is generally recommended when the viscosity ratio $\mu_{l} / \mu_{v}$ is less than 1000 ;

- the Lockhart-Martinelli Correlation (Method) - one of the very first correlations for pressure drop in two-phase flows, proposed by Martinelli and Nelson (1948) and improved by Lockhart and Martinelli (1949);

- the Grönnerud Correlation - a large part of t pressure drop modeling in a two-phase flow was based on neglecting the heat transfer between air and water. In order to remedy this, Grönnerud (1979) developed a correlation based on data obtained from the flow of refrigerants and using an extra correction factor;

- the KRIEGEL method - he investigated the boundary-layer motion of gas and liquid. He calculated the velocity profile in the sheath and in the core in the case of an annular flow, with the hypothesis of a smooth surfaced sheath, and determined the pressure drop based on those data;

- the WALLIS Method - started from the drift model. The two phases flow through two separated cylinders, the cross-sections of which are equal to the total section of the pipe when amounted together. The pressure drop in each cylinder is the real pressure drop of the two-phase flow and can be determined by starting from the single-phase flow and using the average values of velocity;

- the CHAWLA Method - started from the representation of a separate flow of the two phases in a horizontal pipe and calculated the ratio of the velocities of the two phases with the equivalent hydraulic diameter for the cross-section occupied by gas.

Many bibliographical references recommend using the Lockhart-Martinelli correlation (1949) [3]. This method was at first used only in the case of an annular flow of an air-water mixture in a horizontal pipe. Later, it was extended to all domains of flow, as well as to single-component systems and for other substances. Even though there are more precise methods for other domains, this method leads to gratifying results for most of the research conducted up to now ( $\pm 50 \%$ precision). Large deviations can occur in the case of tubing with $D>0,1 \mathrm{~m}$ diameter, at high density flows and at large pressures [4], [5]. Its advantage is its ease of use.

\section{The Lockhart-Martinelli Model}

This model postulates the equality of pressure drops between the two simultaneously flowing phases. These are calculated by taking into account the dimensionless ratio $X$ between the fictive pressure drops for the two phases, which are considered as independently flowing through the pipe.

Therefore, for the section $i-e$, pressure drop on length unit will be determined with the equation:

$$
\left(\frac{\Delta p}{\Delta L}\right)_{i e}=\Phi_{l}^{2}\left(\frac{\Delta p}{\Delta L}\right)_{l}
$$

where:

$$
\begin{aligned}
& \left(\frac{\Delta p}{\Delta L}\right)_{l}=\text { pressure variation on length unit if we assume the liquid flows by itself; } \\
& \Phi_{l}=\text { a dimensionless coefficient that depends on } X \text { and on the structure of the flow. }
\end{aligned}
$$


Further, a set of equations will be developed for the liquid phase. A similar set will be needed for the gas phase, which will be obtained by changing the indices.

The static pressure created by the flow of the liquid, expressed according to the friction factor, is

$$
\left(\frac{\Delta p}{\Delta L}\right)_{i \quad e}=4 f_{l}\left(\frac{\rho_{l}}{d_{l}}\right) \frac{v_{l}^{2}}{2}
$$

where:

$d_{l}=$ hydraulic diameter of the flow of the liquid, [m],

$f_{l}=$ friction factor

$\rho_{l}=$ density of the liquid phase, $\left[\mathrm{kg} / \mathrm{m}^{3}\right]$

$v_{l}=$ average velocity of the liquid phase, $[\mathrm{m} / \mathrm{s}]$

For a more complicated transition section:

$$
A_{l}=\frac{\alpha \pi d_{l}^{2}}{4}
$$

In a similar fashion, the $\beta$ factor is used for gas. For circular sections $\alpha=\beta=1$.

Therefore:

where:

$$
\left(\frac{\Delta \mathrm{p}}{\Delta \mathrm{L}}\right)_{\mathrm{i} \text { e }}=\frac{2 \mathrm{c}_{1} \mathrm{Q}_{\mathrm{M}_{1}}^{2}}{\left(\frac{4 \mathrm{Q}_{\mathrm{M}_{1}}}{\pi \alpha \mathrm{d}_{1} \mu_{1}}\right)^{\mathrm{n}} \alpha^{2} \mathrm{~d}_{1}^{5}\left(\frac{\pi}{4}\right)^{2} \rho_{1}}=\left(\frac{2\left(\frac{4}{\pi}\right)^{2 \mathrm{n}} \mathrm{c}_{1} \mu_{1}^{\mathrm{n}} \mathrm{Q}_{\mathrm{M}_{1}}^{2 \mathrm{n}}}{\mathrm{d}_{0}^{5 \mathrm{n}} \rho_{1}}\right) \alpha^{\mathrm{n} 2}\left(\frac{\mathrm{d}_{\mathrm{o}}}{\mathrm{d}_{1}}\right)^{5 \mathrm{n}}
$$

$c_{l}, n=$ dimensionless coefficients for the liquid phase (tab.1)

$Q_{M_{l}}=$ mass flow of the liquid, $[\mathrm{kg} / \mathrm{s}]$

$\mu_{l}=$ dynamic viscosity of the liquid, [Pa.s]

$d_{0}=$ pipe diameter, $[\mathrm{m}]$

Pressure variation on length unit for liquid is:

$$
\left(\frac{\Delta \mathrm{p}}{\Delta \mathrm{L}}\right)_{1}=\frac{2 \mathrm{f}_{1} \rho_{1} \mathrm{v}_{1}^{2}}{\mathrm{~d}_{0}}=\frac{2 \mathrm{c}_{1} \rho_{1}}{\left(\frac{4 \mathrm{Q}_{\mathrm{M}_{1}}}{\pi \mathrm{d}_{0} \mu_{1}}\right)^{\mathrm{n}}} \frac{\mathrm{Q}_{\mathrm{M}_{1}}^{2}}{\left(\frac{\pi}{4}\right)^{2} \mathrm{~d}_{0}^{4} \rho_{1}^{2} \mathrm{~d}_{0}}=\frac{2\left(\frac{4}{\pi}\right)^{2 \mathrm{n}} \mathrm{c}_{1} \mu_{1}^{\mathrm{n}} \mathrm{Q}_{\mathrm{M}_{1}}^{2 \mathrm{n}}}{\mathrm{d}_{0}^{5}{ }^{\mathrm{n}} \rho_{1}}
$$

From (4), (5) it results that:

$$
\left(\frac{\Delta p}{\Delta L}\right)_{i e}=\left(\frac{\Delta p}{\Delta L}\right)_{l} \alpha^{n 2} 2\left(\frac{d_{0}}{d_{l}}\right)^{5 n}
$$

From equations (6) and (1) is obtained

$$
\frac{\left(\frac{\Delta p}{\Delta L}\right)_{i} e}{\left(\frac{\Delta p}{\Delta L}\right)_{l}}=\Phi_{l}^{2}=\alpha^{n} 2\left(\frac{d_{0}}{d_{l}}\right)^{5 n}
$$


A similar analysis results in corresponding equations for gas (by $m, \beta$ resulting $\Phi_{g}$ ).

The occupation degree of the section with liquid is determined with the equation:

$$
r_{A l}=\alpha \frac{d_{l}^{2}}{d_{0}^{2}}=1 \quad \beta \frac{d_{g}^{2}}{d_{0}^{2}}
$$

From where it results that

$$
\beta=\left(1 \quad r_{A l}\right) \frac{d_{0}^{2}}{d_{g}^{2}}=r_{A g} \frac{d_{0}^{2}}{d_{g}^{2}}
$$

From equations (6), (8) and (9) four variables are obtained $\frac{d_{l}}{d_{0}}, \frac{d_{g}}{d_{0}}, \alpha, \beta$ that can be expressed with the help of four experimental variables $\Phi_{l}, \Phi_{g}, r_{A l}, r_{A g}$.

From equations (1), (5) and (7) the following fraction results

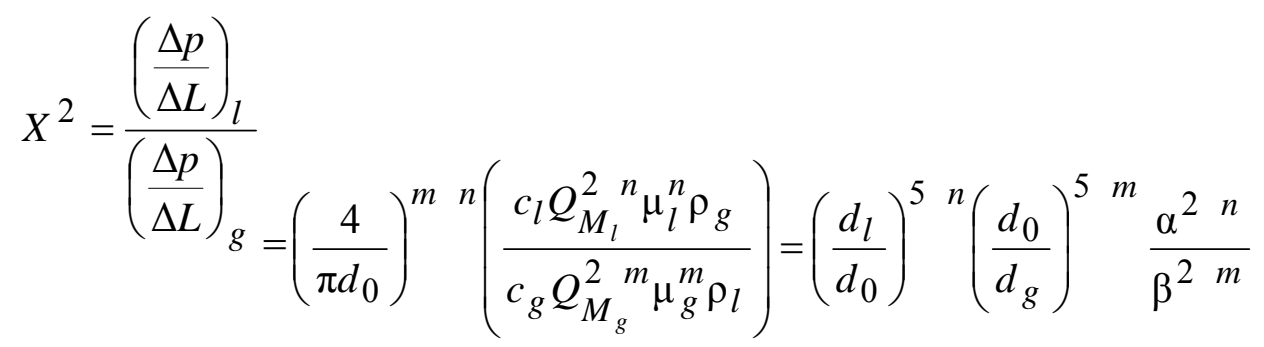

By using a new Reynolds number:

$$
\operatorname{Re}_{l 0}=\frac{4 Q_{M_{l}}}{\pi d_{0} \mu_{l}}
$$

It results that

$$
\frac{\operatorname{Re}_{g 0}^{m}}{\operatorname{Re}_{l 0}^{n}} \frac{c_{l}}{c_{g}} \frac{Q_{M_{l}}^{2}}{Q_{M_{g}}^{2}} \frac{\rho_{g}}{\rho_{l}}=X^{2}
$$

\begin{tabular}{|c|c|c|c|c|c|c|c|c|c|}
\hline \multicolumn{2}{|c|}{$\begin{array}{c}\text { Flow } \\
\text { structure }\end{array}$} & \multicolumn{2}{|c|}{$\mathrm{Re}_{0, i}$} & \multirow{2}{*}{\multicolumn{2}{|c|}{$\begin{array}{c}\text { Indices } \\
{\left[\mathrm{K}_{\mathrm{i}}\right]}\end{array}$}} & \multirow[t]{2}{*}{$c_{l}$} & \multirow[t]{2}{*}{$C_{g}$} & \multirow[t]{2}{*}{$m$} & \multirow[t]{2}{*}{$n$} \\
\hline $\mathrm{L}$ & $\mathrm{G}$ & $\mathrm{L}$ & $G$ & & & & & & \\
\hline$t$ & $t$ & $>2000$ & $>2000$ & $t$ & $t$ & 0,046 & 0,046 & 0,2 & 0,2 \\
\hline$t$ & $l$ & $>2000$ & $<2000$ & $t$ & $l$ & 0,046 & 16 & 1 & 0,2 \\
\hline$l$ & $t$ & $<2000$ & $>2000$ & $l$ & $t$ & 16 & 0,046 & 0,2 & 1 \\
\hline$l$ & $l$ & $<2000$ & $<2000$ & $l$ & $l$ & 16 & 16 & 1 & 1 \\
\hline
\end{tabular}

Therefore $X=f\left(n, m, c_{g}, c_{l}\right)$ coefficients are determined through experimental research, which have highlighted four flow structures, according to table 1.

Table 1

Martinelli's multiplier, coefficients and indices for the calculation of $\boldsymbol{X}$

Note: $t=$ turbulent, $l=$ laminar 
The algorithm for determining the pressure drop for the two-phase gas-liquid flow is:

a) $\operatorname{Re}_{l}, \operatorname{Re}_{g}, f_{l}, f_{g}$ are calculated;

b) $X$ is calculated;

c) by the flow regime and the value of $X, \Phi$ results;

d) $\left(\frac{\Delta p}{\Delta L}\right)_{l, g}$ is calculated;

e) $\left(\frac{\Delta p}{\Delta L}\right)_{i \quad e}=\Phi_{l, g}^{2}\left(\frac{\Delta p}{\Delta L}\right)_{l, g}$ is calculated

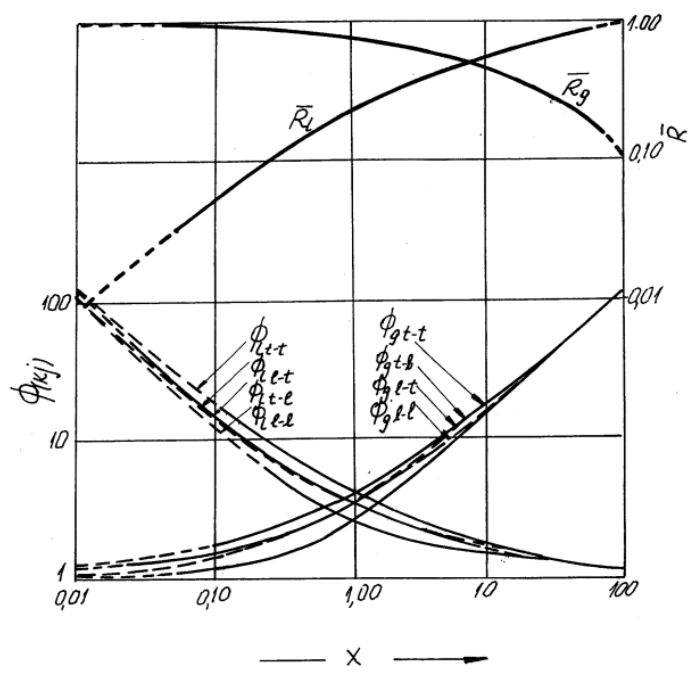

Fig. 1 - Variation curves $\Phi=f(X)$

The variation curves $\Phi=f(X)$ for the flow regimes mentioned in table 1 are resented in figure 1 .

\section{Numerical Simulation of Pressure Drops in a Two-phase Air-water Flow}

On the basis of the algorithm presented in point 3 and the equations (1)-(10), a numerical simulation program for pressure drops in a two-phase flow of fluids through pipes was created. The program was written in FORTRAN. The logic scheme for the calculation program is presented in fig. 2.
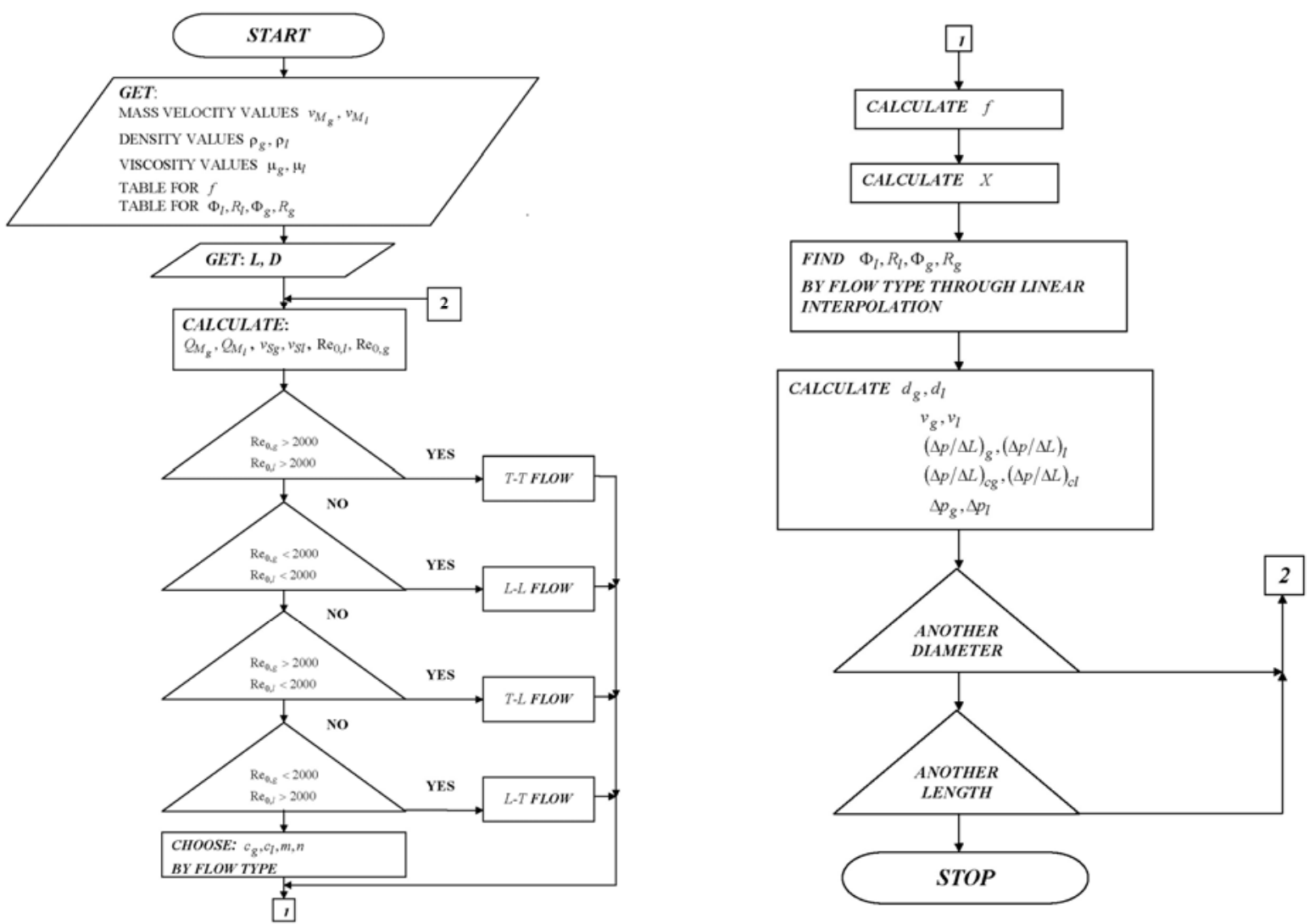

Fig. 2 - The logic scheme of the program (Ro version) 
Values of mass velocities chosen for the numerical simulation

\begin{tabular}{|c|c|c|c|c|c|c|}
\hline$v_{M_{g}}$ & \multicolumn{7}{|c|}{$v_{M_{l}}\left[\mathrm{~kg} /\left(\mathrm{h} \mathrm{m}^{2}\right)\right]$} \\
\hline $\begin{array}{c}\text { Case 1 } \\
336,72\end{array}$ & 329,888 & 989,664 & 3298,88 & 32988,8 & 329888 & 3298880 \\
\hline $\begin{array}{c}\text { Case 2 } \\
3367,2\end{array}$ & $3,29888 \cdot 10^{3}$ & $9,89664 \cdot 10^{3}$ & $32,9888 \cdot 10^{3}$ & $329,888 \cdot 10^{3}$ & $3298,88 \cdot 10^{3}$ & $32988,8 \cdot 10^{3}$ \\
\hline $\begin{array}{c}\text { Case 3 } \\
33672\end{array}$ & $3,29888 \cdot 10^{4}$ & $9,89664 \cdot 10^{4}$ & $32,9888 \cdot 10^{4}$ & $329,888 \cdot 10^{4}$ & $3298,88 \cdot 10^{4}$ & $32988,8 \cdot 10^{4}$ \\
\hline 10101,9 & $3,29888 \cdot 3 \cdot 10^{4}$ & $9,89664 \cdot 3 \cdot 10^{4}$ & $32,9888 \cdot 3 \cdot 10^{4}$ & $329,888 \cdot 3 \cdot 10^{4}$ & $3298,88 \cdot 3 \cdot 10^{4}$ & $32988,8 \cdot 3 \cdot 10^{4}$ \\
\hline $\begin{array}{c}\text { Case 4 } \\
336720\end{array}$ & $3,29888 \cdot 10^{5}$ & $9,89664 \cdot 10^{5}$ & $32,9888 \cdot 10^{5}$ & $329,888 \cdot 10^{5}$ & $3298,88 \cdot 10^{5}$ & $32988,8 \cdot 10^{5}$ \\
\hline
\end{tabular}

For verifying the functionality of the numerical simulation program, an air-water mixture that is to be transported over a length of $152,4 \mathrm{~m}$, through a pipe the diameter of which can have the next values: $d_{0}=0,078 \mathrm{~m} ; 0,102 \mathrm{~m} ; 0,154 \mathrm{~m}$ was used. The mass flows of gas and liquid $Q_{M_{g}}$, $Q_{M_{l}}$ are pairs chosen from the Baker diagram (cases 1,2,3,4). $\Delta p_{c l}$ and $\Delta p_{c g}$ are calculated.

Results as shown in figures $3-8$

\begin{tabular}{|c|c|c|c|}
\hline \multirow{2}{*}{ Figure 3} & Case 1 & $\frac{v_{M_{l}}}{v_{M_{g}}}=676$ & $\Delta p_{c l} \approx \Delta p_{c g}=1,29 \mathrm{kPa}$ \\
\hline & Case 2 & $\frac{v_{M_{l}}}{v_{M_{g}}}=441$ & $\Delta p_{c l} \approx \Delta p_{c g}=43 \mathrm{kPa}$ \\
\hline \multirow{2}{*}{ Figure 4} & Case 3 & $\frac{v_{M_{l}}}{v_{M_{g}}}=441$ & $\Delta p_{c l} \approx \Delta p_{c g}=2700 \mathrm{kPa}$ \\
\hline & Case 4 & $\frac{v_{M_{l}}}{v_{M_{g}}}=441$ & $\Delta p_{c l} \approx \Delta p_{c g}=170581 \mathrm{kPa}$ \\
\hline \multirow{2}{*}{ Figure 5} & Case 1 & $\frac{v_{M_{l}}}{v_{M_{g}}}=576$ & $\Delta p_{c l} \approx \Delta p_{c g}=0,7 \mathrm{kPa}$ \\
\hline & Case 2 & $\frac{v_{M_{l}}}{v_{M_{g}}}=441$ & $\Delta p_{c l} \approx \Delta p_{c g}=31 \mathrm{kPa}$ \\
\hline \multirow{2}{*}{ Figure 6} & Case 3 & $\frac{v_{M_{1}}}{v_{M_{g}}}=441$ & $\Delta p_{c l} \approx \Delta p_{c g}=1960 \mathrm{kPa}$ \\
\hline & Case 4 & $\frac{v_{M_{l}}}{v_{M_{g}}}=441$ & $\Delta p_{c l} \approx \Delta p_{c g}=124000 \mathrm{kPa}$ \\
\hline \multirow{2}{*}{ Figure 7} & Case 1 & $\frac{v_{M_{l}}}{v_{M_{g}}}=484$ & $\Delta p_{c l} \approx \Delta p_{c g}=0,31 \mathrm{kPa}$ \\
\hline & Case 2 & $\frac{v_{M_{l}}}{v_{M_{g}}}=441$ & $\Delta p_{c l} \approx \Delta p_{c g}=19 \mathrm{kPa}$ \\
\hline \multirow{2}{*}{ Figure 8} & Case 3 & $\frac{v_{M_{l}}}{v_{M_{g}}}=441$ & $\Delta p_{c l} \approx \Delta p_{c g}=1200 \mathrm{kPa}$ \\
\hline & Case 4 & $\frac{v_{M_{l}}}{v_{M_{g}}}=441$ & $\Delta p_{c l} \approx \Delta p_{c g}=76300 \mathrm{kPa}$ \\
\hline
\end{tabular}


The results obtained were transposed in the diagrams in figures $3,4,5,6,7,8$.

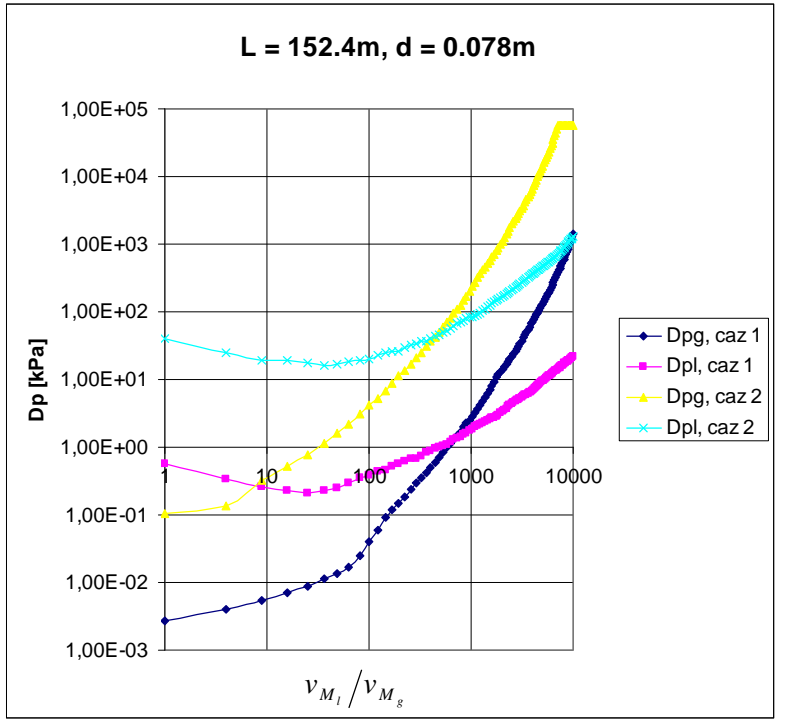

Fig. 3

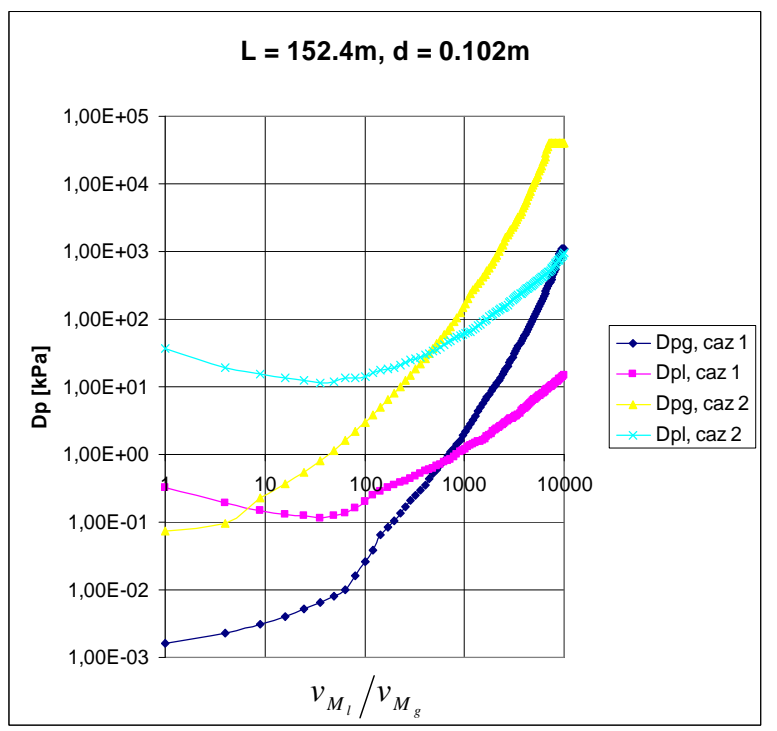

Fig. 5

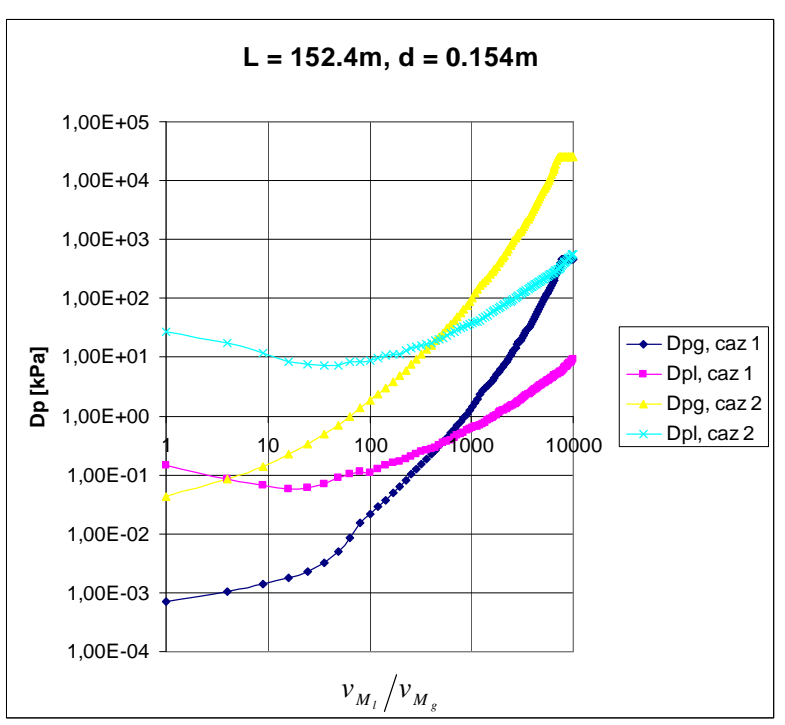

Fig. 7

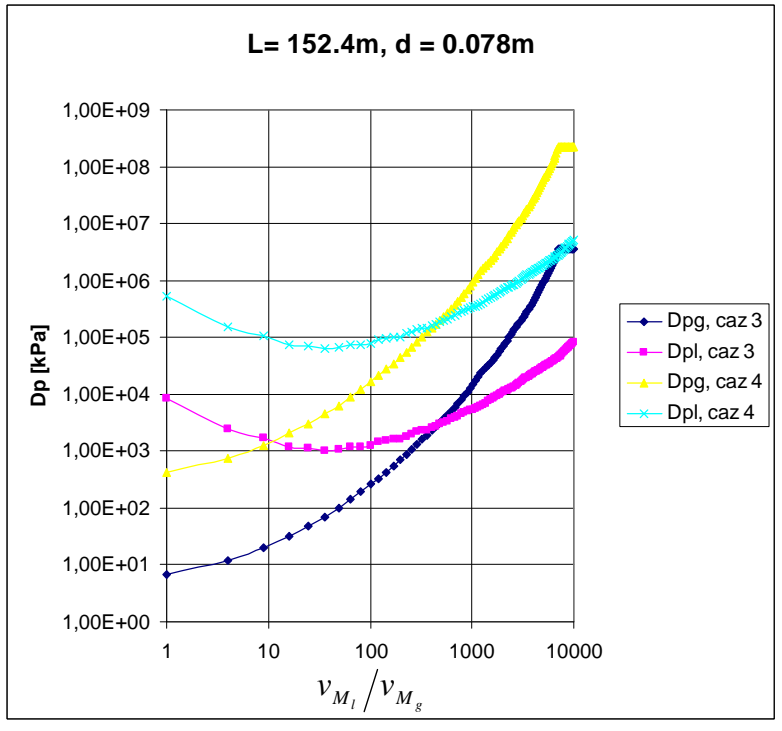

Fig. 4

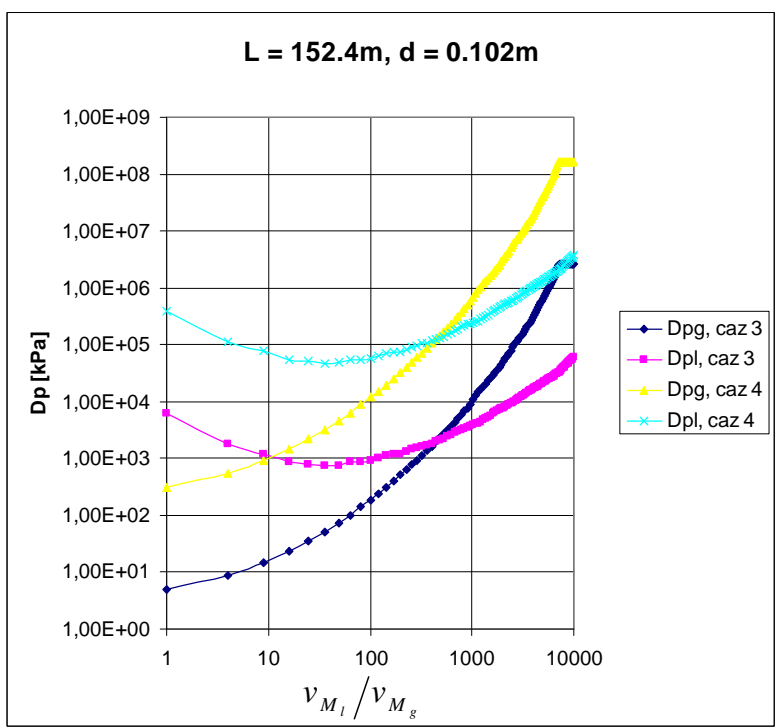

Fig. 6

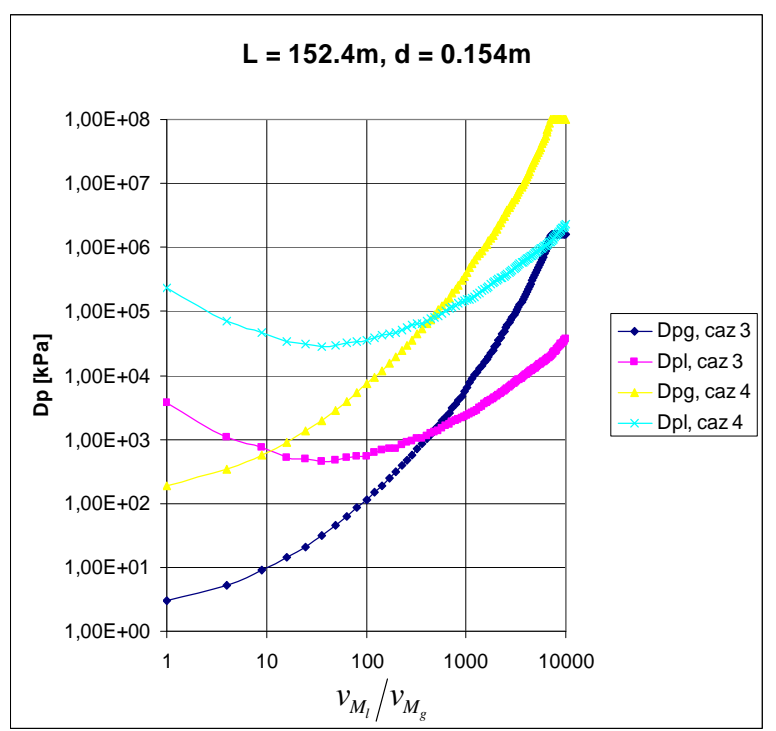

Fig. 8 
It can be observed that the calculation model Lockhart-Martinelli is valid for $\frac{v_{M_{l}}}{v_{M_{g}}} \in\left[10^{2} \ldots 10^{3}\right]$.

\section{Conclusions}

The elaborate calculation program is based on the Lockhart-Martinelli method and is easily usable for reproducing an entire range of practical calculation situations. Referring to the Lockhart-Martinelli method it can be ascertained that the hypotheses that are at its basis are verified in the fraction $\frac{v_{M_{l}}}{v_{M_{g}}}$ between $10^{2}$ and $10^{3}$, mainly consisting of bubbly and liquid burst flows. For these types of flow, $\Delta p_{l} \approx \Delta p_{g}$.

\section{References}

[1] ASHRAE. (2009). Handbook-Fundamentals.

[2] M. B. Ould-Didi, N. Kattan, and J. R. Thome. (2002). Prediction of two-phase gradients of refrigerants in horizontal tubes. Int. J. Refrigeration, 25, 935-947.

[3] R. W. Lockhart and R. C. Martinelli. (1949). Proposed correlation of data for isothermal two-phase, twocomponent in pipes. Chem. Eng. Process, 45(1), 39-48.

[4] R. S. Brodkey. (1976). The phenomena of fluid motions. Dover publications, New York.

[5] O. Baker. (1954). Design of pipe lines for simultaneous flow of oil and gas. Oil and Gas J., 53, 185-190. 